\title{
CONDIÇÕES PARA BIESTABILIDADE EM FREQUÊNCIA DE LASER SEMICONDUTOR SOB REALIMENTAÇÃO ÓTICA ORTOGONAL
}

\author{
Samuel B. Alves ${ }^{1}$, Martine CheVrollier ${ }^{1}$, Thierry Passerat De Silans ${ }^{1}$, Hugo L. D. De S. CAVAlCAN- \\ TE $^{1,2}$, MARCOS ORIÁ ${ }^{1}$ \\ ${ }^{1}$ Laboratório de Física Atômica e Lasers, D.F., Universidade Federal da Paraíba, Cx. Postal 5086, 58051- \\ 970 João Pessoa-PB, Brasil \\ ${ }^{2}$ Departamento de Informática, Universidade Federal da Paraíba, 58051-970 João Pessoa-PB, Brasil \\ samuel@otica.ufpb.br
}

\begin{abstract}
Resumo- Biestabilidade na frequência de emissão de um laser semicondutor sujeito a realimentação ótica com polarização ortogonal foi observada experimentalmente por Farias et al. em 2005. Um modelo de equações de taxa para esse sistema dinâmico, apresentado posteriormente por Masoller et al. em 2007, que leva em conta os efeitos térmicos e ganho de saturação, prevê uma variação linear da frequência do laser com a intensidade do campo de realimentação. Nesse trabalho, usando o mesmo modelo, estudamos o processo de biestabilidade ótica em frequência nesses sistemas com realimentação filtrada, determinando as condições espectrais do filtro de realimentação necessárias para o aparecimento de histerese que leva a biestabilidade.
\end{abstract}

Palavras-chave— Laser semicondutor, realimentação ótica, sistema dinâmico, biestabilidade em frequência, histerese.

\section{I - Introdução}

Realimentação é o mecanismo pelo qual parte do sinal de saída de um sistema é transferida para a entrada deste mesmo sistema. A realimentação é um importante mecanismo de controle cujas aplicações se dão em várias áreas do conhecimento [1,2]. Cavidades óticas são sistemas físicos adaptados ao estudo da realimentação, onde a biestabilidade é um dos comportamentos mais importantes derivado da realimentação em sistemas não-lineares [3]. A biestabilidade em cavidades óticas [4] tem sido observada quando ambas as respostas dispersiva [5] e absorsiva [6] do meio não linear são exploradas.

Lasers semicondutores são também largamente usados para estudos de resposta dinâmica da radiação emitida quando estes sistemas não-lineares estão na presença de realimentação [7,8]. Como conseqüência, lasers de diodo (DL) têm sido utilizados, por exemplo, para investigar biestabilidade em potência [9] e em polarização [10].

Em nosso laboratório estudou-se a biestabilidade em frequência [11] usando realimentação ortogonal filtrada. $\mathrm{O}$ filtro sensível à frequência é colocado no caminho do feixe de retorno para modular a potência de realimentação, em função da frequência de emissão laser. Foram observadas então duas frequências de emissão para uma mesma potência de saída (as variações de amplitude são menores que $1 \%$ ).

Biestabilidade em amplitude apresenta potenciais aplicações em portas lógicas do tipo 'tudo óptico' e em computação óptica, além de outras aplicações. A biestabilidade na frequência abre caminho para aplicações onde a informação digital pode ser realizada com um nível de intensidade constante, porém entre dois estados de frequências diferentes. As observações de biestabilidade em frequência foram descritas [12] em termos de um modelo que leva em conta a forma de linha do filtro e a relação linear do deslocamento em frequência induzido com a potência de realimentação. Contudo, uma descrição deta- lhada da resposta espectral do filtro ainda não foi realizada, de forma a determinar sob quais condições podemos observar biestabilidade na frequência ótica.

Neste trabalho, usando um modelo para as equações de taxa de lasers semicondutores [12] e levando em conta a largura de linha e acoplamento efetivo da realimentação, nós determinamos sob quais condições é possível observar biestabilidade na frequência ótica do laser semicondutor.

Simulações numéricas usando tal descrição podem reproduzir o comportamento dinâmico observado experimentalmente. Além disso, observamos a não existência de biestabilidade quando usamos filtros espectralmente muito largos na mesma configuração experimental descrita em [11].

Este trabalho é organizado da seguinte forma: Na seção II nós descrevemos a montagem experimental e caracterizamos o filtro espectral usado em nossas medidas. Na seção III apresentamos o modelo teórico que prevê as condições para a existência de biestabilidade ótica em frequência e mostramos os resultados para a simulação numérica usando esse modelo. Mostramos, na quarta seção, que usando um filtro espectral com largura de linha suficientemente grande a biestabilidade ótica em frequência não se manifesta para os níveis de intensidade de realimentação disponíveis, como observado em [11]. São apresentadas, na seção $\mathrm{V}$, as conclusões e perspectivas do presente trabalho.

\section{II - Descrição experimental e caracterização do filtro espectral}

Um laser de diodo (DL) monomodo, estabilizado em corrente e temperatura, emitindo em torno de $780 \mathrm{~nm}$, é colocado em uma configuração que permite devolver parte do feixe de saída do laser de volta na cavidade do DL. A configuração experimental (Figura 1) é essencialmente a mesma descrita em [11], onde o filtro atômico é substituído por uma grade de difração. Seguindo o caminho ótico, a radi- 
ação laser incide diretamente na grade de difração, de onde o modo de ordem $m=1$ é alinhado para realimentação. Esse feixe atravessa uma lâmina de meia onda que gira a polarização do campo e, por fim, um polarizador Glan-Foucault. Esse polarizador também permite que a luz laser ortogonalmente polarizada retorne na cavidade laser. $O$ conjunto lâmina de onda e polarizador permite o controle da intensidade reinjetada. Um divisor de feixe pré-calibrado colocado no caminho da realimentação nos permite determinar a intensidade da radiação enviada na cavidade pela realimentação ótica ortogonal. Parte do sinal laser é enviado através de uma lente que focaliza o feixe no centro de uma abertura micrométrica (pinhole, $5 \mu \mathrm{m}$ ) medindo a curva de transmissão do filtro como função da frequência.

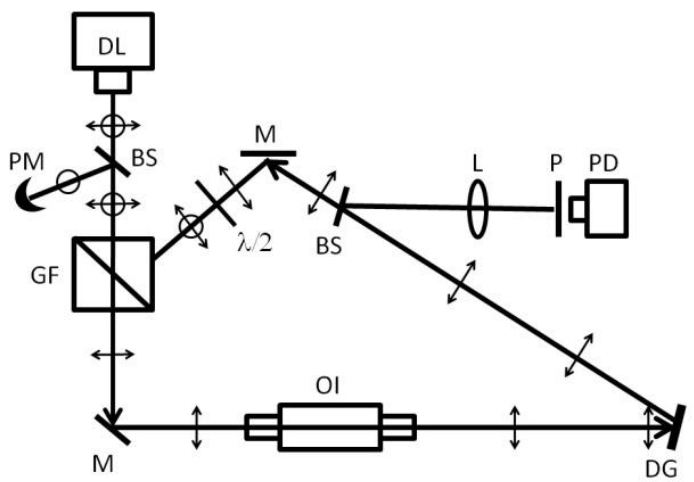

Figura 1: Montagem experimental para a medida da curva de transmissão. DL: laser de diodo, GF: Polarizador Glan-Foucault, M: Espelho, BS: Divisor de Feixe, OI: Isolador Ótico, $\lambda / 2$ : Lâmina de meia onda, P: Pinhole, L: Lente, PD: Fotodetector, PM: Medidor de Potência, $\longleftrightarrow$ Laser com polarização paralela ao plano do arranjo experimental, $\odot$ Laser com polarização perpendicular ao plano do arranjo experimental.

Esse sistema de detecção e análise nos permite estudar modificações na forma de linha em função da realimentação.

A Figura 2 mostra a curva de transmissão normalizada como função da frequência para uma grade de difração de 1800 linhas/mm, usando-se um pinhole de $5 \mu \mathrm{m}$ para simular a entrada da cavidade do semicondutor.

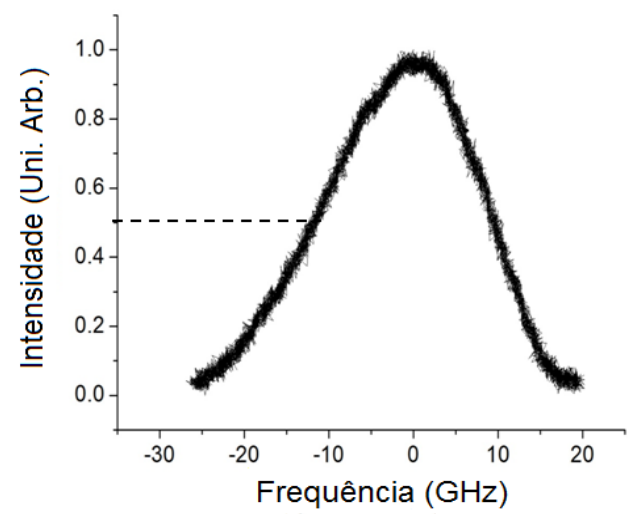

Figura 2: Curva de transmissão normalizada como função da frequência para uma grade de difração de 1800 linhas $/ \mathrm{mm}$.

Como podemos observar na Figura 2 a "forma de linha" da grade de difração é bem mais larga (cer- ca de $20 \mathrm{GHz}$ ) do que a de uma transição atômica (tipicamente de $1 \mathrm{GHz}$ de largura Doppler), que gera a biestabilidade observada em [11].

\section{III - Analise teórica e simulação}

Para analisarmos analiticamente a resposta do laser à filtragem espectral vamos fazer uma aproximação de sua forma de linha por uma curva Gaussiana. A potência que retorna ao laser pode ser então escrita na forma:

$$
P_{f}(v)=\kappa_{0} \exp \left(-\alpha\left(v-v_{\text {cent }}\right)^{2}\right) P,
$$

onde $\kappa_{0}$ é a fração da potência de saída que retorna na cavidade na ausência do filtro espectral, o fator $\alpha$ está associado à largura a meia altura da forma de linha, $v$ é a frequência de emissão do laser e $v_{\text {cent }}$ é a frequência central de transmissão da grade, ou seja, onde a realimentação é máxima. A potência reinjetada na cavidade laser provoca um deslocamento $\delta$ na frequência de emissão deste [11]:

$$
\delta=v_{s o l}-v=\beta P_{f}(v)
$$

$\operatorname{com} v_{\text {sol }}$ a frequência do laser sem realimentação e $\beta$ um coeficiente de proporcionalidade característico do laser. A partir das equações (1) e (2), obtemos:

$$
v_{\text {sol }}-v_{\text {cent }}=v-v_{\text {cent }}+\beta \kappa_{0} \exp \left(-\alpha\left(v-v_{\text {cent }}\right)^{2}\right) P \text {. }
$$

Definindo-se $x=v-v_{\text {cent }}$ e $y=v_{\text {sol }}-v_{\text {cent }}$ as mudanças esperadas na forma de linha (aparecimento da histerese) ocorrem quando os valores para a derivada $d x / d y$ ficam negativos [13]. Os valores para que essa condição seja satisfeita são dados pela equação:

$$
\begin{gathered}
1-2 \alpha \beta \kappa_{0} P x \exp \left(-\alpha x^{2}\right)<0, \\
2 \alpha \beta \kappa_{0} P x>\exp \left(\alpha x^{2}\right) .
\end{gathered}
$$

O surgimento da histerese ocorre quando:

$$
2 \alpha \beta \kappa_{0} P>\sqrt{2 \alpha} \exp \left(\frac{1}{2}\right)
$$

que é obtido quando resolvemos para $x$ a equação (5).

Usando nessa analise teórica $\alpha=1,0 \times$ $10^{-8} \mathrm{MHz}^{-2}$ (valor típico para a forma de linha da grade de difração), temos $\beta \kappa_{0} P=11658 \mathrm{MHz}$, ou seja, para termos efeitos perceptíveis (histerese particularmente) na emissão do laser devemos ter $\beta \kappa_{0} P \approx 1,2 \times 10^{4} \mathrm{MHz}$, para este valor do parâmetro $\alpha$. As curvas mostradas nas Figura 3, 4 e 5 simulam o perfil de transmissão com realimentação usando três valores diferentes para $\beta \kappa_{0} P$, ou seja, três níveis diferentes de realimentação, usando $\alpha=1,0 \times$ $10^{-8} \mathrm{MHz}^{-2}$. 


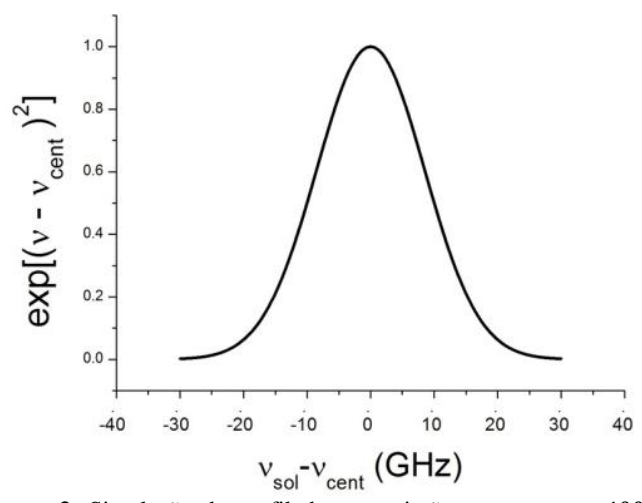

Figura 3: Simulação do perfil de transmissão para $\mathrm{MHz}^{-2}$

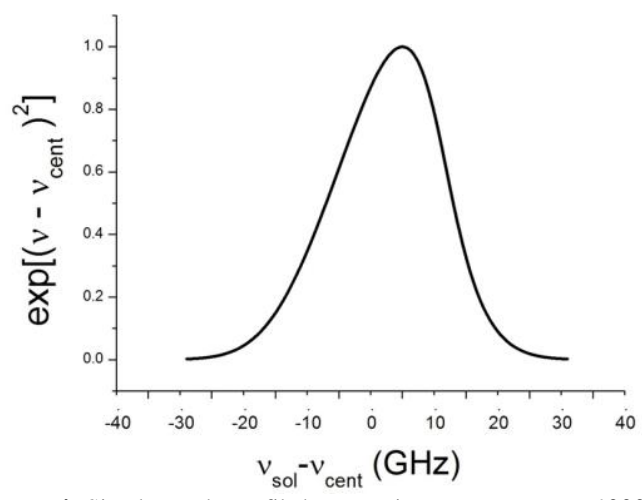

Figura 4: Simulação do perfil de transmissão para $\mathrm{MHz}^{-2}$

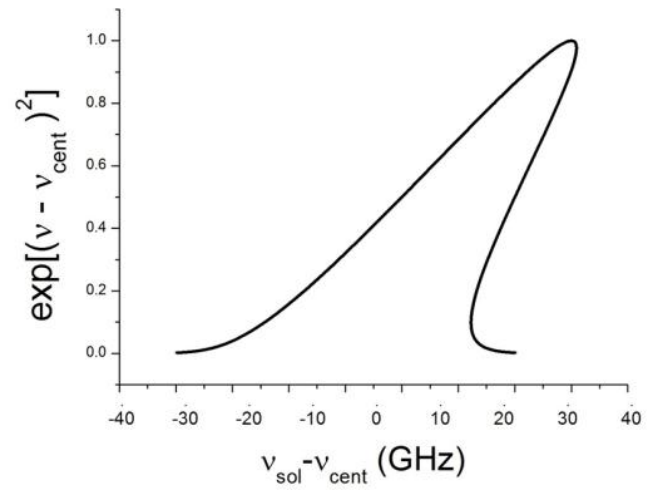

Figura 5: Simulação do perfil de transmissão para $\mathrm{MHz}$ e $\mathrm{MHz}^{-2}$

\section{IV - Resultados experimentais}

As Figuras 6 e 7 mostram as curvas de transmissão da luz laser através do sistema de análise ('pinhole' + detector) com e sem realimentação, respectivamente. Na Figura 7 podemos observar um deslocamento da curva de transmissão devido à resposta linear da frequência do semicondutor como função da potência de realimentação.

Medidas do deslocamento em frequência em função da potência de realimentação não filtrada, para esse laser, permitiram obter o coeficiente de proporcionalidade $\beta=1,08 \pm 0,05 \mathrm{GHz} / \mathrm{mW}$.

Medimos (Figuras 6 e 7) um deslocamento em frequência de aproximadamente $\delta=7,4 \mathrm{GHz}$, que nos leva a uma potência de realimentação de $\mathrm{mW}$.

A Figura 2 mostra a curva de transmissão normalizada e a largura a meia altura dessa curva pode ser estimada em $21 \mathrm{GHz}$, que nos leva a

Os efeitos da biestabilidade (mudança nos flancos da forma de linha) aparecem se a condição dada pela equação (6) for satisfeita. Usando-se o valor obtido em (7), temos:

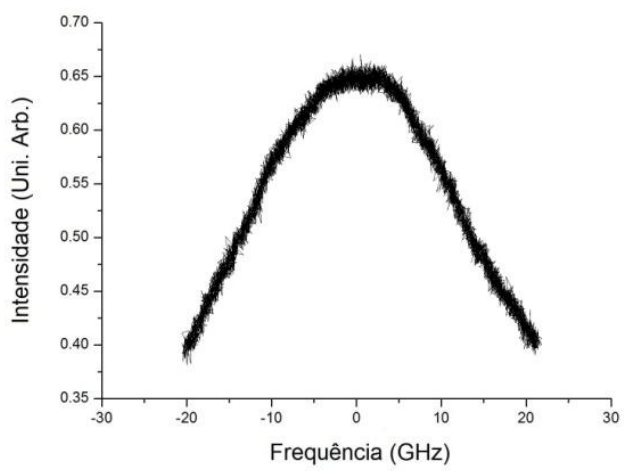

Figura 6: Curva de transmissão da grade de difração sem realimentação.

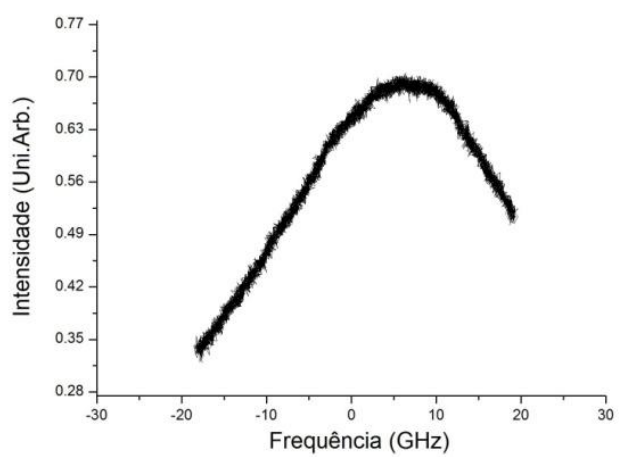

Figura 7: Curva de transmissão da grade de difração com realimentação. As curvas aparecem "truncadas", pois para obter as curvas de transmissão a amplitude da varredura deveria ser alta (filtro espectral largo) e para maiores amplitude na modulação o laser passava a apresentar comportamento indesejado (saltos de modos de frequência).

Por outro lado sabemos que

$093 \mathrm{GHz}$, desta forma a condição necessária para que os efeitos de histerese desejados possam existir não é satisfeita. A modificação na curva de transmissão do filtro é perceptível, porém sem histerese. Sendo assim, os efeitos não podem ser observados experimentalmente como mostra a curva da Figura 7. De fato, como vimos na análise teórica da seção anterior, precisamos de muito mais potência de rea- 
limentação para utilizar esse filtro, que é espectralmente muito largo.

\section{V - Conclusão}

Neste trabalho nós utilizamos uma grade de difração como filtro espectral para analisar o comportamento espectral de um laser semicondutor sob realimentação ótica ortogonal. A motivação para o uso deste filtro é a sua largura espectral, bem maior que a largura espectral de uma linha atômica estudada anteriormente.

Determinamos analiticamente sob quais condições temos biestabilidade ótica em frequência quando submetemos um laser semicondutor à realimentação ortogonal filtrada e mostramos que a largura de linha do filtro, assim como o nível de intensidade de realimentação disponível são fatores determinantes para o aparecimento de biestabilidade.

Experimentalmente, observamos que usando na realimentação uma grade de difração a modificação da forma de linha da emissão laser é apenas parcial, sem ciclo de histerese. Esse resultado é compatível com nosso modelo que prevê níveis de intensidade muito maiores que o disponível para se obter biestabilidade em frequência com este filtro espectralmente largo.

\section{Agradecimentos}

Agradecemos ao CNPq, CAPES e FINEP pelo suporte financeiro.

\section{Referências Bibliográficas}

[1] S. Serizawa, K. Miyamichi, H. Nakatani, M. Suzuki, M. Saito, Y. Yoshihara, and H. Sakano, "Negative feedback regulation ensures the one receptor-one olfactory neuron rule in mouse", Science 302, 2088 (2003). DOI: 10.1126/science. 1089122

[2] S. Grill, V. S. Zykov, and S. C. Müller, J. Phys. Chem. 100, 19082 (1996). DOI: 10.1021/jp9616674

[3] L. A. Lugiato, in Progress in Optics, edited by E. Wolf (North-Holland, Amsterdam, 1984), Vol. 21, p. 71 .

[4] H. M. Gibbs, Optical Bistability: Controlling Light with Light (Academic, New York, 1985).

[5] H. M. Gibbs, S. L. McCall, and T. N. C. Venkatesan, "Differential Gain and Bistability Using a Sodium-Filled Fabry-Perot Interferometer"Phys. Rev. Lett. 36, 1135 (1976). DOI:

10.1103/PhysRevLett.36.1135

[6] A. T. Rosenberger, L.A. Orozco, and H. J. Kimble, "Observation of absorptive bistability with two- level atoms in ring cavity" Phys. Rev. A 28, R2569 (1983). DOI: 10.1103/PhysRevA.28.2569

[7] H. Kawaguchi, Bistabilities and Nonlinearities in Laser Diodes (Artech House, Boston, 1994).

[8] G. P. Agrawal and N. K. Dutta, Long-Wavelength Semiconductor Lasers (Van Nostrand Reinhold, New York, 1986). DOI: 10.1007/978-94-011-6994-3

[9] P. Pakdeevanich and M. J. Adams, "Switching powers for optical bistability in a semiconductor laser above and below threshold" Opt. Commun. 176, 195 (2000). DOI: 10.1016/S0030-4018(00)00512-5

[10] Y. C. Chen and J. M. Liu, "Polarization bistability in semiconductor lasers" Appl. Phys. Lett. 46, 16 (1985); DOI: 10.1063/1.95834

[11] S. Cecchi, G. Giusfredi, E. Petriella, and P. Salieri, "Observation of Optical Tristability in So-dium Vapors" Phys. Rev. Lett. 49, 1928 (1982). DOI: 10.1103/PhysRevLett.49.1928

[12]B. Farias, T. P. de Silans, M. Chevrollier, and M. Oriá, "Frequency bistability of a semiconductor laser under a frequency-dependent feedback," Phys. Rev. Lett., vol. 94, 173902 (2005). DOI: 10.1103/PhysRevLett.94.173902

[13]C. Masoller, T. Sorrentino, M. Chevrollier, and M. Oria, "Bistability in Semiconductor Lasers With Polarization-Rotated Frequency-Dependent Optical Feedback" IEEE J. Quantum Electronics, vol. 43, 261 ( 2007) DOI: 10.1109/JQE.2006.889647

[14] Eugene Hecht, 'Optics' $2^{\text {nd }}$ edition, (AddisonWesley Pub. Co. 1987) p. 426. 